\title{
Erratum to: Environmental Orientation of Exporting SMEs from an Emerging Economy: Its Antecedents and Consequences
}

\author{
Ricky Y. K. Chan ${ }^{1} \cdot$ Katherine H. Y. $\mathrm{Ma}^{2}$
}

Published online: 9 June 2016

(C) Springer-Verlag Berlin Heidelberg 2016

\section{Erratum to: Manag Int Rev \\ DOI 10.1007/s11575-016-0280-0}

Due to formatting problems some columns in Tables 4 and 5 were not in the right order.

The original article was corrected and updated.

Please excuse this mistake.

The online version of the original article can be found under doi:10.1007/s11575-016-0280-0.

Ricky Y. K. Chan

msricky@polyu.edu.hk

1 Department of Management and Marketing, Hong Kong Polytechnic University, Hung Hom, Kowloon, Hong Kong

2 Youth College (Kwai Fong Campus), Vocation Training Council, Kwai Chung, New Territories, Hong Kong 
Table 4 Results of multiple regression analysis (direct effects)

$\begin{array}{llll}\text { Model 1 } & \text { Model 2 } & \text { Model 3 } & \text { Model 4 } \\ \text { Internal } & \text { External } & \text { Proactive } & \text { Corporate } \\ \text { environmental } & \text { environmental } & \text { environmental } & \text { export } \\ \text { orientation (IEO) } & \text { orientation (EEO) } & \text { strategies (PES) } & \begin{array}{l}\text { performance } \\ \text { (CEP) }\end{array}\end{array}$

\begin{tabular}{|c|c|c|c|c|}
\hline \multicolumn{5}{|l|}{ Controls } \\
\hline Firm size (SIZE) & -0.02 & 0.03 & 0.01 & 0.11 \\
\hline Firm age (AGE) & 0.02 & 0.02 & -0.04 & 0.03 \\
\hline $\begin{array}{l}\text { International } \\
\text { experience (IE) }\end{array}$ & 0.03 & 0.05 & 0.01 & 0.01 \\
\hline $\begin{array}{l}\text { Foreign shareholding } \\
\quad \text { (FS) }\end{array}$ & 0.11 & 0.06 & 0.03 & 0.02 \\
\hline Industry type (IND) & 0.07 & $0.14^{*}$ & 0.10 & -0.06 \\
\hline $\begin{array}{l}\text { Number of exporting } \\
\text { ventures (NEV) }\end{array}$ & 0.01 & $0.14^{*}$ & 0.09 & 0.07 \\
\hline $\begin{array}{l}\text { Social desirability bias } \\
\quad \text { (SDB) }\end{array}$ & 0.08 & 0.02 & 0.08 & 0.01 \\
\hline \multicolumn{5}{|l|}{ Direct effects } \\
\hline $\begin{array}{l}\text { CEO environmental } \\
\text { beliefs (CEOEB) }\end{array}$ & $0.32 * *$ & & & \\
\hline $\begin{array}{l}\text { SMEs' efforts in } \\
\text { scanning developed } \\
\text { markets (SESDM) }\end{array}$ & & $0.22 *$ & & \\
\hline $\begin{array}{l}\text { Internal environmental } \\
\text { orientation (IEO) }\end{array}$ & & & $0.22 * *$ & \\
\hline $\begin{array}{c}\text { External environmental } \\
\text { orientation (EEO) }\end{array}$ & & & $0.49 * *$ & \\
\hline $\begin{array}{l}\text { Proactive } \\
\text { environmental } \\
\text { strategies (PES) }\end{array}$ & & & & $0.32 * *$ \\
\hline Model F statistic & $11.11 * *$ & $9.54 * *$ & $24.17 * *$ & $6.90 * *$ \\
\hline Degree of freedom & 8 & 8 & 9 & 8 \\
\hline Adjusted $\mathrm{R}^{2}$ & 0.18 & 0.16 & 0.35 & 0.12 \\
\hline Summarized results & Support $\mathrm{H}_{1}$ & Support $\mathrm{H}_{2}$ & $\begin{array}{l}\text { Support } \mathrm{H}_{3} \text { and } \\
\mathrm{H}_{4}\end{array}$ & Support $\mathrm{H}_{5}$ \\
\hline
\end{tabular}

All reported estimates are standardized regression coefficients

$* * p<0.01 ; * p<0.05$ 
Table 5 Results of multiple regression analysis (moderating effects)

\begin{tabular}{|c|c|c|c|c|}
\hline & $\begin{array}{l}\text { Model } 5 \\
\text { Proactive } \\
\text { environmental } \\
\text { strategies (PES) }\end{array}$ & $\begin{array}{l}\text { Model } 6 \\
\text { Proactive } \\
\text { environmental } \\
\text { strategies (PES) }\end{array}$ & $\begin{array}{l}\text { Model } 7 \\
\text { Corporate export } \\
\text { performance } \\
(\mathrm{CEP})\end{array}$ & $\begin{array}{l}\text { Model } 8 \\
\text { Corporate export } \\
\text { performance } \\
(\mathrm{CEP})\end{array}$ \\
\hline \multicolumn{5}{|l|}{ Controls } \\
\hline Firm size (SIZE) & 0.01 & 0.01 & 0.11 & 0.08 \\
\hline Firm age (AGE) & -0.04 & -0.04 & 0.03 & 0.03 \\
\hline $\begin{array}{l}\text { International } \\
\text { experience (IE) }\end{array}$ & 0.01 & 0.01 & 0.01 & 0.02 \\
\hline $\begin{array}{l}\text { Foreign } \\
\quad \text { shareholding (FS) }\end{array}$ & 0.03 & 0.01 & 0.02 & 0.01 \\
\hline Industry type (IND) & 0.10 & 0.08 & -0.06 & -0.03 \\
\hline $\begin{array}{l}\text { Number of exporting } \\
\text { ventures (NEV) }\end{array}$ & 0.08 & 0.07 & 0.07 & 0.07 \\
\hline $\begin{array}{l}\text { Social desirability } \\
\text { bias (SDB) }\end{array}$ & 0.08 & 0.08 & 0.01 & 0.01 \\
\hline \multicolumn{5}{|l|}{ Direct effects } \\
\hline $\begin{array}{l}\text { Internal } \\
\quad \text { environmental } \\
\text { orientation (IEO) }\end{array}$ & $0.22 * *$ & $0.14 *$ & & \\
\hline $\begin{array}{l}\text { External } \\
\text { environmental } \\
\text { orientation (EEO) }\end{array}$ & $0.49 * *$ & $0.42 * *$ & & \\
\hline $\begin{array}{l}\text { Proactive } \\
\text { environmental } \\
\text { strategies (PES) }\end{array}$ & & & $0.32 * *$ & $0.30 *$ \\
\hline $\begin{array}{l}\text { Local ecological } \\
\text { infrastructure } \\
\text { (LEI) }\end{array}$ & 0.05 & 0.06 & 0.02 & 0.02 \\
\hline \multicolumn{5}{|l|}{ Interaction effects } \\
\hline LEI $\times$ IEO & & 0.11 & & \\
\hline LEI $\times$ EEO & & $0.36 * *$ & & \\
\hline LEI $\times$ PES & & & & $0.22 * *$ \\
\hline Model F statistic & $21.70 * *$ & $25.21 * *$ & $6.12 * *$ & $11.37 * *$ \\
\hline Degree of freedom & 10 & 12 & 9 & 10 \\
\hline Adjusted $\mathrm{R}^{2}$ & 0.35 & 0.43 & 0.12 & 0.22 \\
\hline Summarized results & & $\begin{array}{l}\text { Support } \mathrm{H}_{7} \text { but not } \\
\mathrm{H}_{6}\end{array}$ & & Support $\mathrm{H}_{8}$ \\
\hline
\end{tabular}

All reported estimates are standardized regression coefficients

** $p<0.01 ; * p<0.05$ 